\title{
QUADRATIC SYSTEMS WITH INVARIANT STRAIGHT LINES OF TOTAL MULTIPLICITY TWO HAVING DARBOUX INVARIANTS
}

\author{
JAUME LLIBRE AND REGILENE D. S. OLIVEIRA
}

\begin{abstract}
In this paper we present the global phase portraits in the Poincaré disc of the planar quadratic polynomial systems which admit invariant straight lines with total multiplicity two and Darboux invariants.
\end{abstract}

\section{INTRODUCTION}

Let $\mathbb{R}[x, y]$ be the ring of the real polynomials in the variables $x$ and $y$. We consider the system of differential equations in $\mathbb{R}^{2}$ defined by

$$
\begin{aligned}
& \dot{x}=P(x, y), \\
& \dot{y}=Q(x, y),
\end{aligned}
$$

where $P, Q \in \mathbb{R}[x, y]$. The dot denotes derivative with respect to the time $t$. We say that $m=\max \{\operatorname{deg} P, \operatorname{deg} Q\}$ is the degree of system (1).

A quadratic polynomial differential system or simply a quadratic system is a system (1) for which $m=2$. This type of differential equations appear in the modeling of many natural phenomena described in different branches of science, in biological and physical applications. Besides the applications the quadratic systems became a matter of interest for the mathematicians. Now more than one thousand papers have been published about quadratic systems, see for instance [7] for a bibliographical survey.

In this paper we always assume that the polynomials $P$ and $Q$ are coprime. Otherwise system (1) can be reduced to a linear or constant system doing a rescaling of the time variable, and we want only to study quadratic systems.

The knowledge of the first integrals is of particular interest in planar differential systems because they allow to draw their phase portraits. On the other hand it is also interesting to know if the differential system has invariants, i.e. first integrals depending on the time. Roughly speaking, the invariants that here we will study (the Darboux invariants) allow to describe the asymptotic behavior of the solutions of the system, as we shall see later on in Proposition 5.

Key words and phrases. quadratic vector fields, Darboux invariant, phase portraits. 2010 Mathematics Subject Classification: Primary 34C05, 34A34. 


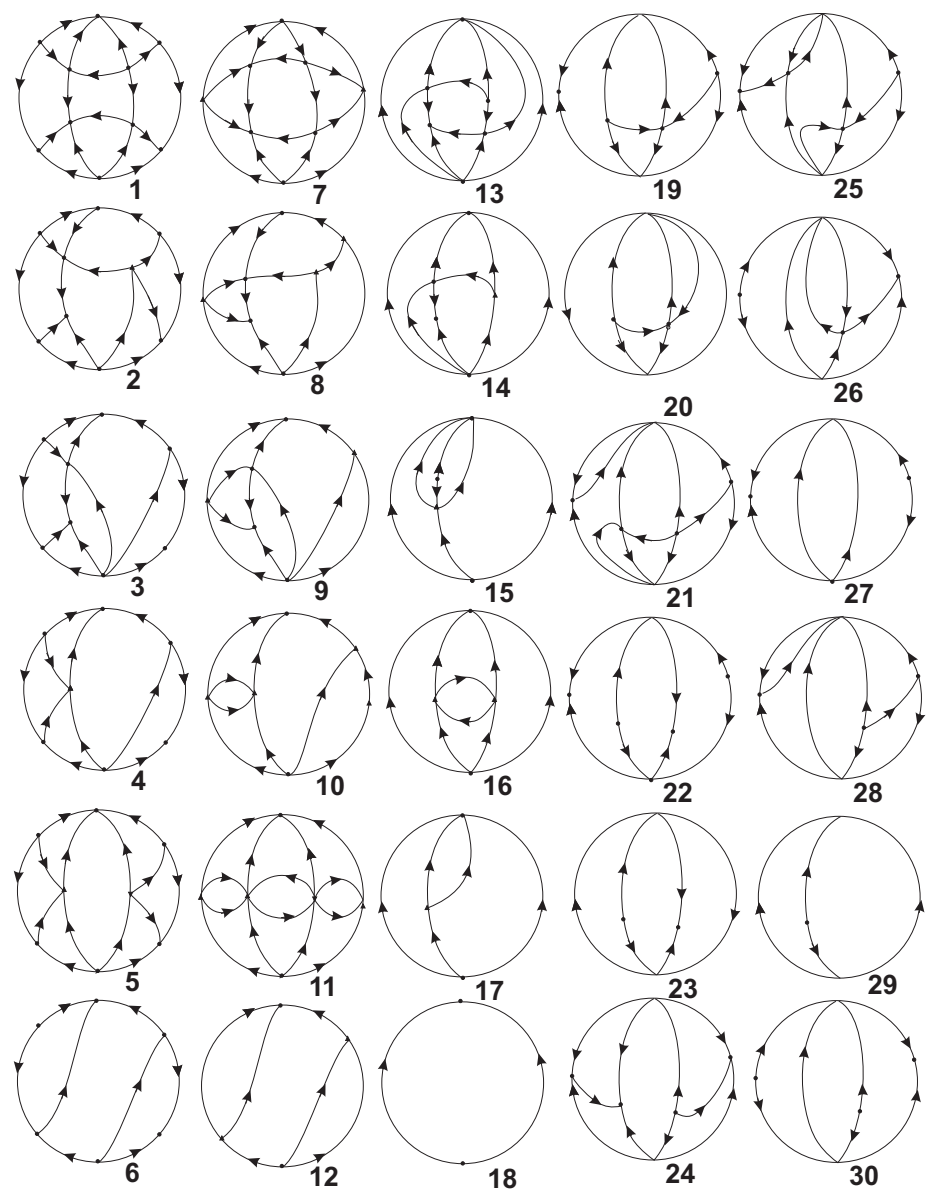

Figure 1. All global phase portraits of systems of type $(R P L)$.

In this paper we investigated quadratic systems which admit real or complex invariant straight lines of total multiplicity two and having a special invariant of the form $f(x, y) e^{s t}$, called Darboux invariant defined in the subsection 2.2 .

Given two real or complex straight lines in the plane they could be parallels, intersecting at a point, or coincide (a double line). So we shall say that a quadratic system is of type $(R P L)$ if it has two real and parallel straight lines. In similar way, the system is of type $(C P L)$ if it has two complex and parallels straight lines; it is of type $(L V)$ if it has two real straight lines intersecting at a real point (Lotka-Volterra systems); or of type $(p)$ if it has two complex straight lines intersecting at a real point $(p)$, finally, it is of type $(D L)$ if it has one double invariant real straight line.

The normal forms of all quadratic systems with two real or complex invariant straight lines is given in the next proposition proved in [2]. 


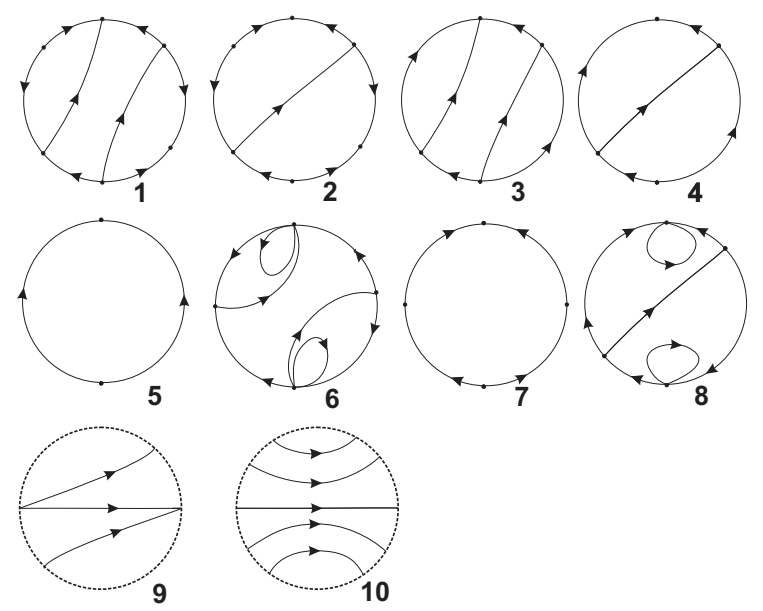

Figure 2. Global phase portraits of systems of type $(C P L)$.

Proposition 1. Applying an affine change of coordinates the real quadratic systems, having two real or complex invariant straight lines taken into account their multiplicities, can be written in one of the following five forms:

$$
\begin{aligned}
& (R P L) \quad \dot{x}=x^{2}-1, \quad \dot{y}=Q(x, y), \\
& (C P L) \quad \dot{x}=x^{2}+1, \quad \dot{y}=Q(x, y), \\
& (D L) \quad \dot{x}=x^{2}, \quad \dot{y}=Q(x, y), \\
& (L V) \quad \dot{x}=x(a x+b y+c), \quad \dot{y}=y(A x+B y+C), \\
& \text { (p) } \quad \dot{x}=\frac{a}{2}\left(x^{2}+y^{2}\right)+\frac{c}{2} x+2 y(p x+q y+r), \\
& \dot{y}=\frac{b}{2}\left(x^{2}+y^{2}\right)+\frac{c}{2} y-2 x(p x+q y+r),
\end{aligned}
$$

where $Q(x, y)=d+a x+b y+l x^{2}+m x y+n y^{2}$ denotes an arbitrary polynomial of degree 2 .

Our first main result given in the next theorem is the characterization of the Darboux invariants of the quadratic systems having two complex or real invariant straight lines taking into account their multiplicities.

Theorem 2. Quadratic systems of Proposition 1 having a Darboux invariant are:

$(R P L)$ all systems of type $(R P L)$ have the Darboux invariant

$$
I_{1}(x, y, t)=\sqrt{x+1} e^{t} / \sqrt{x-1} .
$$

$(C P L)$ all systems of type $(C P L)$ have the Darboux invariant

$$
I_{2}(x, y, t)=e^{\arctan (1 / x)} e^{t} .
$$




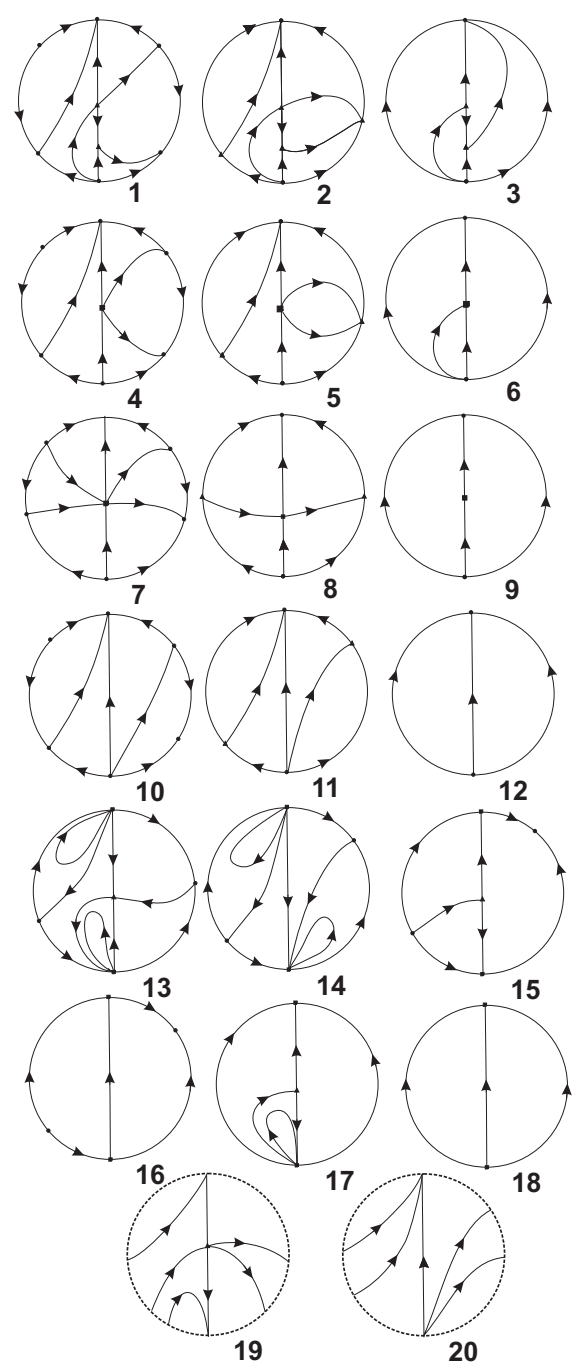

FiguRE 3. Global phase portraits of systems of type $(D L)$.

$(D L)$ all systems of type $(D L)$ have the Darboux invariant

$$
I_{3}(x, y)=e^{(1 / x)} e^{t} .
$$

$(L V)$ Systems of type $(L V)$ which have a Darboux invariant can be written either as

$$
\dot{x}=x(a x+b y+c), \quad \dot{y}=C y \quad \text { with } C \neq 0,
$$

or as

(3) $\dot{x}=x\left(-A \frac{\lambda_{2}}{\lambda_{1}} x-B \frac{\lambda_{2}}{\lambda_{1}} y+c\right), \quad \dot{y}=y(A x+B y+C) \quad$ with $\lambda_{1} \neq 0$, 
where $\lambda_{1}$ and $\lambda_{2}$ are constants, having Darboux invariants

$$
I_{4}(x, y, t)=y e^{-C t} \quad \text { and } \quad I_{5}(x, y, t)=x^{\lambda_{1}} y^{\lambda_{2}} e^{\left(-c \lambda_{1}-C \lambda_{2}\right) t},
$$

respectively. This statement is proved in [1].

$(p)$ Systems of type $(p)$ which have a Darboux invariant can be written either as

$$
\begin{aligned}
& \dot{x}=c x+4 y(p x+q y+r), \\
& \dot{y}=c y-4 x(p x+q y+r),
\end{aligned}
$$

where $c^{2}+p^{2}+q^{2} \neq 0$ having Darboux invariant

$$
I_{6}(x, y, t)=\left(x^{2}+y^{2}\right) e^{-c t}
$$

or as

$$
\begin{aligned}
& \dot{x}=a\left(x^{2}+y^{2}\right)+c x+y((b-a) x-(a+b v) y+2 r), \\
& \dot{y}=b\left(x^{2}+y^{2}\right)+c y-x((b-a) x-(a+b v) y+2 r),
\end{aligned}
$$

with $\left(a^{2}+b^{2}\right) v \neq 0$, having a Darboux invariant given by

Our second main result provides the global classification of all phase portraits in the Poincaré disc of the quadratic systems with two complex or real invariant straight lines taking into account their multiplicities and having a Darboux invariant.

Theorem 3. The phase portrait on the Poincaré disc of any quadratic polynomial differential system

$(R P L)$ with two real and parallel invariant straight lines is topologically equivalent to one of the 30 phase portraits described in Figure 1;

$(C P L)$ with two complex parallel invariant straight lines is topologically equivalent to one of the 10 phase portraits described in Figure 2;

$(D L)$ with a double invariant straight line is topologically equivalent to one of the 20 phase portraits described in Figure 3;

$(L V)$ with two real invariant straight lines intersecting in a point given by systems (2) and (3) are topologically equivalent to one of the 31 phase portraits described in [1];

$\left(p_{1}\right)$ with two complex invariant straight line intersecting in a real point given by system (4) is topologically equivalent to the phase portrait described in Figure 4; and

$\left(p_{2}\right)$ with two complex invariant straight line intersecting in a real point given by system (5) is topologically equivalent to one of the 4 phase portraits described in Figure 5.

The paper is organized as follows. Basic definitions and results necessary to complete the study of the Darboux invariants and of the phase portraits of the quadratic systems are given in section 2 . In sections 3 and 4 we prove Theorems 2 and 3 respectively. 


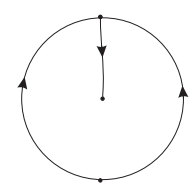

Figure 4. Global phase portraits of systems of type $(p)$ given by system (4).

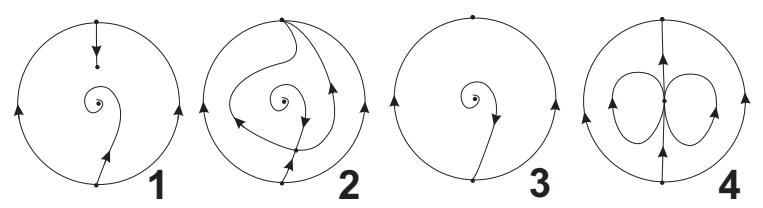

Figure 5. Global phase portraits of systems of type $(p)$ given by system (5).

\section{BASIC RESULTS}

The main of this section is introduce some definitions and results which shall be used in next sections for the study of the Darboux invariants and to obtain the local phase portraits of finite and infinite singularities of system (1).

2.1. Invariants. Let $U$ be an open and dense subset of $\mathbb{R}^{2}$, we say that a nonconstant function $H: U \rightarrow \mathbb{R}$ is a first integral of system (1) on $U$ if $H(x(t), y(t))$ is constant for all of the values of $t$ for which $(x(t), y(t))$ is a solution of system (1) contained in $U$. Obviously $H$ is a first integral of system (1) if and only if

$$
P \frac{\partial H}{\partial x}+Q \frac{\partial H}{\partial y}=0
$$

for all $(x, y) \in U$.

An invariant of system (1) on the open subset $U$ of $\mathbb{R}^{2}$ is a nonconstant analytic function $I$ in the variables $x, y$ and $t$ such that $I(x(t), y(t), t)$ is constant on all solution curves $(x(t), y(t))$ of system (1) contained in $U$, i.e.

$$
\frac{\partial I}{\partial x} P+\frac{\partial I}{\partial y} Q+\frac{\partial I}{\partial t}=0
$$

for all $(x, y) \in U$. In short, $I$ is a first integral of system (1) depending on the time $t$.

On the other hand given $f \in \mathbb{C}[x, y]$ we say that the curve $f(x, y)=0$ is an invariant algebraic curve of system (1) if there exists $K \in \mathbb{C}[x, y]$ such that

$$
P \frac{\partial f}{\partial x}+Q \frac{\partial f}{\partial y}=K f
$$


The polynomial $K$ is called the cofactor of the invariant algebraic curve $f=0$. When $K=0, f$ is a polynomial first integral. When a real polynomial differential system has a complex invariant algebraic curve, then it has also its conjugate. It is important to consider the complex invariant algebraic curves of the real systems because sometimes these forces the real integrability of the system, for more details see Chapter 8 of [5], or the subsection 2.2 .

Let $f, g \in \mathbb{C}[x, y]$ and assume that $f$ and $g$ are relatively prime in the ring $\mathbb{C}[x, y]$, or that $g=1$. Then the function $\exp (f / g)$ is called a exponential factor of system (1) if for some polynomial $L \in \mathbb{C}[x, y]$ of degreee at most $m-1$ we have

$$
P \frac{\partial \exp (f / g)}{\partial x}+Q \frac{\partial \exp (f / g)}{\partial y}=L \exp (f / g)
$$

As before we say that $L$ is the cofactor of the exponential factor $\exp (f / g)$. We observe that in the definition of exponential factor $\exp (f / g)$ if $f, g \in$ $\mathbb{C}[x, y]$ then the exponential factor is a complex function. Again when we look for a complex exponential factor of a real polynomial system we are thinking the real polynomial system as a complex polynomial system.

2.2. Darboux invariants. An invariant $I$ is called a Darboux invariant if it can be written as

$$
I(x, y, t)=f_{1}^{\lambda_{1}} \cdots f_{p}^{\lambda_{p}} F_{1}^{\mu_{1}} \cdots F_{q}^{\mu_{q}} e^{s t},
$$

where $f_{i}=0$ are invariant algebraic curves of system (1) for $i=1, \ldots p$, and $F_{j}$ are exponential factors of system (1) for $j=1, \ldots, q, \lambda_{i}, \mu_{j} \in \mathbb{C}$ and $s \in \mathbb{R} \backslash\{0\}$.

Observe that if among the invariant algebraic curves a complex conjugate pair $f=\operatorname{Re}(f)+\operatorname{Im}(f) i=0$ and $\bar{f}=\operatorname{Re}(f)-\operatorname{Im}(f) i=0$ occurs, then the Darboux invariant has a factor of the form $f^{\lambda} \bar{f}^{\lambda}$, which is the real multivalued function $\left((\operatorname{Re}(f))^{2}+(\operatorname{Im}(f))^{2}\right)^{\operatorname{Re}(\lambda)} e^{-2 \operatorname{Im}(\lambda) \arctan (\operatorname{Im}(f) / \operatorname{Re}(f))}$. So if system (1) is real then the Darboux invariant is also real, independently of the fact of having complex invariant curves or complex exponential factors.

The next result, proved in [3], explain how to find Darboux invariants.

Proposition 4. Suppose that a polynomial system (1) of degree $m$ admits $p$ invariant algebraic curves $f_{i}=0$ with cofactors $K_{i}$ for $i=1, \ldots, p, q$ exponential factors $\exp \left(g_{j} / h_{j}\right)$ with cofactors $L_{j}$ for $j=1, \ldots, q$, then, if there exist $\lambda_{i}$ and $\mu_{j} \in \mathbb{C}$ not all zero such that

$$
\sum_{i=1}^{p} \lambda_{i} K_{i}+\sum_{j=1}^{q} \mu_{j} L_{j}=-s
$$


for some $s \in \mathbb{R} \backslash\{0\}$, then substituting $f_{i}^{\lambda_{i}}$ by $\left|f_{i}\right|^{\lambda_{i}}$ if $\lambda_{i} \in \mathbb{R}$, the real (multivalued) function

$$
f_{1}^{\lambda_{1}} \ldots f_{p}^{\lambda_{p}}\left(\exp \left(\frac{g_{1}}{h_{1}}\right)\right)^{\mu_{1}} \ldots\left(\exp \left(\frac{g_{q}}{h_{q}}\right)\right)^{\mu_{q}} e^{s t}
$$

is a Darboux invariant of system (1).

Moreover, the search of first integrals is a classic tool in order to describe the phase portraits of a 2-dimensional differential system. As usual the phase portrait of a system is the decomposition of the domain of definition of this system as union of all its orbits.

Let $\phi_{p}(t)$ be the solution of system (1) passing through the point $p \in \mathbb{R}^{2}$, defined on its maximal interval $\left(\alpha_{p}, \omega_{p}\right)$ such that $\phi_{p}(0)=p$. If $\omega_{p}=\infty$ we define the set

$$
\omega(p)=\left\{p \in \mathbb{R}^{2}: \exists\left\{t_{n}\right\} \text { with } t_{n} \rightarrow \infty \text { and } \phi_{p}\left(t_{n}\right) \rightarrow q \text { when } n \rightarrow \infty\right\} .
$$

In the same way, if $\alpha_{p}=-\infty$ we define the set

$$
\alpha(p)=\left\{p \in \mathbb{R}^{2}: \exists\left\{t_{n}\right\} \text { with } t_{n} \rightarrow-\infty \text { and } \phi_{p}\left(t_{n}\right) \rightarrow q \text { when } n \rightarrow \infty\right\} .
$$

The sets $\omega(p)$ and $\alpha(p)$ are called the $\omega$-limit set (or simply $\omega$-limit) and the $\alpha$-limit set (or $\alpha$-limit) of $p$, respectively. For more details on the $\omega$ and $\alpha$-limit sets see for instance section 1.4 of [5].

The Darboux invariant provides information about the $\omega$ - and $\alpha$-limit sets of all orbits of system (1). More precisely, we have the following result, where the definition of Poincaré compactification and Poincaré disc is given in subsection 2.3.

Proposition 5. Let $I(x, y, t)=f(x, y) e^{\text {st }}$ be a Darboux invariant of system (1). Let $p \in \mathbb{R}^{2}$ and $\phi_{p}(t)$ the solution of system (1) with maximal interval $\left(\alpha_{p}, \omega_{p}\right)$ such that $\phi_{p}(0)=p$.

(1) If $\omega_{p}=\infty$ then $\omega(p) \subset\{f(x, y)=0\} \cup \mathbb{S}^{1}$,

(2) If $\alpha_{p}=-\infty$ then $\alpha(p) \subset\{f(x, y)=0\} \cup \mathbb{S}^{1}$.

Here $\mathbb{S}^{1}$ denotes the infinity of the Poincaré disc.

Proof. We prove statement (1), the proof of statement (2) is similar. Suppose $s>0$ and let $\phi_{p}(t)=\left(x_{p}(t), y_{p}(t)\right)$. Since $I(x, y, t)$ is an invariant $I\left(x_{p}(t), y_{p}(t), t\right)=a \in \mathbb{R}$ for all $t \in\left(\alpha_{p}, \omega_{p}\right)$. Then

$$
a=\lim _{t \rightarrow \infty} I\left(x_{p}(t), y_{p}(t), t\right)=\lim _{t \rightarrow \infty} f\left(x_{p}(t), y_{p}(t)\right) e^{s t} .
$$

As $\lim _{t \rightarrow \infty} e^{s t}=\infty$, we have that $\lim _{t \rightarrow \infty} f\left(x_{p}(t), y_{p}(t)\right)=0$. So, by continuity and the definition of $\omega$-limit set it follows that $\omega(\mathrm{p}) \subset\{f(x, y)=0\}$, and for the $\alpha$-limit set $\alpha(\mathrm{p}) \in \mathbb{S}^{1}$. 


\subsection{Poincaré compactification. Let}

$$
\mathcal{X}=P(x, y) \frac{\partial}{\partial x}+Q(x, y) \frac{\partial}{\partial y}
$$

be the planar polynomial vector field of degree $m$ associated to the polynomial differential system (1). The Poincaré compactified vector field $\pi(\mathcal{X})$ corresponding to $\mathcal{X}$ is an analytic vector field induced on $\mathbb{S}^{2}$ as follows (for more details, see [5]).

Let $\mathbb{S}^{2}=\left\{y=\left(y_{1}, y_{2}, y_{3}\right) \in \mathbb{R}^{3} ; y_{1}^{2}+y_{2}^{2}+y_{3}^{2}=1\right\}$ and $T_{y} \mathbb{S}^{2}$ be the tangent plane to $\mathbb{S}^{2}$ at point $y$. We identify $\mathbb{R}^{2}$ with $T_{(0,0,1)} \mathbb{S}^{2}$ and we consider the central projection $f: T_{(0,0,1)} \mathbb{S}^{2} \rightarrow \mathbb{S}^{2}$. The map $f$ defines two copies of $\mathcal{X}$ on $\mathbb{S}^{2}$, one in the southern hemisphere and the other in the northern hemisphere. Denote by $\mathcal{X}^{\prime}$ the vector field $D(f \circ \mathcal{X})$ defined on $\mathbb{S}^{2} \backslash \mathbb{S}^{1}$, where $\mathbb{S}^{1}=\left\{y \in \mathbb{S}^{2} ; y_{3}=0\right\}$, identified with the infinity of $\mathbb{R}^{2}$.

For extending $\mathcal{X}^{\prime}$ to a vector field on $\mathbb{S}^{2}$, including $\mathbb{S}^{1}, \mathcal{X}$ must satisfy convenient conditions. Since the degree of $\mathcal{X}$ is $m, \pi(\mathcal{X})$ is the unique analytic extension of $y_{3}^{m-1} \mathcal{X}^{\prime}$ to $\mathbb{S}^{2}$. On $\mathbb{S}^{2} \backslash \mathbb{S}^{1}$ there is two symmetric copies of $\mathcal{X}$, and once we know the behavior of $\pi(\mathcal{X})$ near $\mathbb{S}^{1}$, we know the behavior of $\mathcal{X}$ in a neighborhood of the infinity. The Poincaré compactification has the property that $\mathbb{S}^{1}$ is invariant under the flow of $\pi(\mathcal{X})$. The projection of the closed northern hemisphere of $\mathbb{S}^{2}$ on $y_{3}=0$ under $\left(y_{1}, y_{2}, y_{3}\right) \mapsto\left(y_{1}, y_{2}\right)$ is called the Poincaré disc, and its boundary is $\mathbb{S}^{1}$.

Two polynomial vector fields $\mathcal{X}$ and $\mathcal{Y}$ on $\mathbb{R}^{2}$ are topologically equivalent if there exists a homeomorphism on $\mathbb{S}^{2}$ preserving the infinity $\mathbb{S}^{1}$ carrying orbits of the flow induced by $\pi(\mathcal{X})$ into orbits of the flow induced by $\pi(\mathcal{Y})$ preserving or not the orientation of the orbits.

As $\mathbb{S}^{2}$ is a differentiable manifold, in order to compute the explicit expression of $\pi(\mathcal{X})$, we consider 6 local charts $U_{i}=\left\{y \in \mathbb{S}^{2} ; y_{i}>0\right\}$ and $V_{i}=\left\{y \in \mathbb{S}^{2} ; y_{i}<0\right\}$, where $i=1,2,3$, and the diffeomorphisms $F_{i}: U_{i} \rightarrow \mathbb{R}^{2}$ and $G_{i}: V_{i} \rightarrow \mathbb{R}^{2}$, for $i=1,2,3$, which are the inverses of the central projections from the tangent planes at the points $(1,0,0)$, $(-1,0,0),(0,1,0),(0,-1,0),(0,0,1)$ and $(0,0,-1)$, respectively. We denote by $z=(u, v)$ the value of $F_{i}(y)$ and $G_{i}(y)$, for any $i=1,2,3$, therefore $z$ means different things depending on the local charts that we are working. So, after some computations, $\pi(\mathcal{X})$ is given by:

$$
\begin{gathered}
v^{m} \Delta(z)\left(Q\left(\frac{1}{v}, \frac{u}{v}\right)-u P\left(\frac{1}{v}, \frac{u}{v}\right),-v P\left(\frac{1}{v}, \frac{u}{v}\right)\right) \text { in } U_{1}, \\
v^{m} \Delta(z)\left(P\left(\frac{u}{v}, \frac{1}{v}\right)-u Q\left(\frac{u}{v}, \frac{1}{v}\right),-v Q\left(\frac{u}{v}, \frac{1}{v}\right)\right) \text { in } U_{2}, \\
\Delta(z)(P(u, v), Q(u, v)) \text { in } U_{3},
\end{gathered}
$$


where $\Delta(z)=\left(u^{2}+v^{2}+1\right)^{-(m-1) / 2}$. The expressions for $V_{i}$ 's are the same as that for $U_{i}$ 's but multiplied by the factor $(-1)^{m-1}$. In these coordinates $v=0$ always denotes the points of $\mathbb{S}^{1}$.

\section{DARBoux inVARIANTS}

In order to prove Theorem 2 we investigate each system described in Proposition 1 which provides the normal forms of all quadratic system with two real or complex invariant straight lines taking into account their multiplicities.

Proof of statement (RPL) of Theorem 2. Let $f_{1}=x-1$ and $f_{2}=x+1$. It is easy to check that $f_{1}$ and $f_{2}$ satisfy the equation

$$
\frac{\partial f_{i}}{\partial x} \dot{x}+\frac{\partial f_{i}}{\partial y} \dot{y}=f_{i} K_{i}
$$

where $K_{i}=f_{j}, i \neq j, i, j=1,2$. So $f_{1}=0$ and $f_{2}=0$ are invariant straight lines with cofactors $K_{1}$ and $K_{2}$, respectively.

Moreover, as the equation $\lambda_{1} K_{1}+\lambda_{2} K_{2}+s=0$ has the solution $\lambda_{1}=$ $-\lambda_{2}=s / 2$, it follows from Proposition 4 that $I_{1}(x, y, t)=\sqrt{x+1} e^{s t} / \sqrt{x-1}$ is a Darboux invariant for the systems of the type $(R P L)$ for any $s \in \mathbb{R} \backslash\{0\}$. Choosing $s=1$ we have the Darboux invariant of the statement $(R P L)$ of Theorem 2 .

Proof of statement (CPL) of Theorem 2. The proof of this statement is very similar to the proof of statement $(R P L)$. Using the same arguments we can prove that $f_{1}=x-i$ and $f_{2}=x+i$ are invariant curves for systems of type $(C P L)$ with cofactors $K_{1}=x+i$ and $K_{2}=x-i$, respectively. Moreover the solution of equation $\lambda_{1} K_{1}+\lambda_{2} K_{2}+s=0$ is $\lambda_{1}=-i s / 2, \lambda_{2}=i s / 2$ for any $s \neq 0$. So $I_{2}(x, y, t)=(x-i)^{-\frac{i s}{2}}(x+i)^{\frac{i s}{2}} e^{s t}$ is the Darboux invariant for any system of type $(C P L)$ for every $s \in \mathbb{R} \backslash\{0\}$. Using the polar form of the complex numbers it follows that $(x-i)^{-i / 2}(x+i)^{i / 2}=e^{\arctan (1 / x)}$. So choosing $s=1$ we can rewrite the Darboux invariant as $I_{2}(x, y, t)=$ $e^{\arctan (1 / x)+t}$ and this statement is proved.

Proof of statement (DL) of Theorem 2. As $f_{1}=x$ satisfies the equation $\frac{\partial f_{1}}{\partial x} \dot{x}+\frac{\partial f_{1}}{\partial y} \dot{y}=f_{1} x, f_{1}=x$ is an invariant straight line for systems of type $(D L)$ with cofactor $K=x$. From the definition of exponential factor it follows that $f_{2}=e^{1 / x}$ is an exponential factor for systems of type $(D L)$ of cofactor $L=-1$. Moreover, the unique solution of $\lambda K+\mu L+s=0$ is $\lambda=0$ and $\mu=s$, so $I_{3}(x, y, t)=e^{s / x+s t}$ is a Darboux invariant for systems of type $(D L)$ for any $s \neq 0$. Taking $s=1$ we conclude the proof of this statement.

For systems of type $(R P L),(C P L)$ and $(D L)$ there are no restrictions for the existence of Darboux invariants. When the quadratic systems have two invariant straight lines (real or complex) intersecting at a real point there 
are restrictions. This means that there are systems of type $(L V)$ and of type $(p)$ which do not admit Darboux invariants.

Proof of the statement ( $p$ ) of Theorem 2. It is easy to check that $f_{1}=x+i y$ and $f_{2}=x-i y$ satisfy equations (9) with $K_{1}=\frac{1}{2}((c+a x+b y)+i(4 r+4 p x+$ $4 q y+a y-b x)$ ) and $K_{2}=\bar{K}_{1}$, respectively, so $f_{1}=0$ and $f_{2}=0$ are algebraic invariant straight lines and, $K_{1}, K_{2}$ their cofactors. Since $K_{2}$ is the conjugate of $K_{1}$ and $s$ is a real number, $\lambda_{1}$ and $\lambda_{2}$ in the equation $\lambda_{1} k_{1}+\lambda_{2} k_{2}+s=0$ must satisfy $\lambda_{2}=\overline{\lambda_{1}}$. In this way the Darboux invariant will be real as already mentioned. The solutions of equation $\lambda_{1} k_{1}+\lambda_{2} k_{2}+s=0$ could be divided in two. If $\lambda_{1}=\lambda_{2} \in \mathbb{R}$ then $a=b=0$ and $s=-\lambda_{1} c$, so the Darboux invariant is $I_{6}(x, y, t)=\left(x^{2}+y^{2}\right)^{\lambda_{1}} e^{-c t \lambda_{1}}$, for all $\lambda_{1}$ nonzero, in particular $\lambda_{1}=1$. Otherwise, writting $\lambda_{1}=u+i v$ the solution is $p=(b-a u) / 4 v, q=-(b u+a v) / 4 v, s=-c u-4 r v$ and the Darboux invariant is $I_{7}(x, y, t)=\left(x^{2}+y^{2}\right)^{u} e^{-2 v \arctan (v / u)-(c u+4 r v) t}$ with $v \neq 0$. Fixing $u=1$ and rescaling the time by a factor $4 v$, the statement $(p)$ of Theorem 2 is proved.

\section{Phase Portraits}

In this section we prove Theorem 3 .

Proof of statement (RPL) of Theorem 3. In order to study systems of type $(R P L)$ we considere two cases.

Case 1: $n \neq 0$. In this case there are 18 non-equivalent phase portraits in the Poincaré disc. Indeed after applying an affine change of variables we can fix $n=1$ in systems of type $(R P L)$. For studying the singular points at infinity of a polynomial vector field via the Poincaré compactification we need to study the singular points in the local chart $U_{1}$ and the origin of the local chart $U_{2}$.

In the chart $U_{1}$ the compactified system of type $(R P L)$ with $n=1$, writes

$$
\begin{aligned}
& \dot{z}_{1}=l-z_{1}+m z_{1}+z_{1}^{2}+a z_{2}+b z_{1} z_{2}+d z_{2}^{2}+z_{1} z_{2}^{2}, \\
& \dot{z}_{2}=\left(-1+z_{2}\right) z_{2}\left(1+z_{2}\right) .
\end{aligned}
$$

We define $\delta=1-4 l-2 m+m^{2}$ and the points

$$
p^{ \pm}=\left(z_{1}, z_{2}\right)=((1-m \pm \sqrt{\delta}) / 2,0) .
$$

If $\delta>0$ then $p^{+}$is a hyperbolic saddle point and $p^{-}$is a hyperbolic stable node, see more details Theorem 2.15 of [5]. If $\delta=0$ then $p^{+}=p^{-}$and the unique infinite singular point in the chart $U_{1}$ is a semi-hyperbolic singular point. It follows from Theorem 2.19 of [5] that this is a saddle-node point. Finally, if $\delta<0$ then there are no infinite singular points in the chart $U_{1}$.

Moreover, systems of type $(R P L)$ in the chart $U_{2}$ are written as

$$
\begin{aligned}
& \dot{z_{1}}=-z_{1}+z_{1}^{2}-m z_{1}^{2}-l z_{1}^{3}-b z_{1} z_{2}-a z_{1}^{2} z_{2}-z_{2}^{2}-d z_{1} z_{2}^{2}, \\
& \dot{z_{2}}=-z_{2}\left(1+m z_{1}+l z_{1}^{2}+b z_{2}+a z_{1} z_{2}+d z_{2}^{2}\right),
\end{aligned}
$$


having the origin as a hyperbolic stable node.

Now we shall study the finite singular points. Denote by $d^{-}=4(-a+$ $d+l)+(b-m)^{2}$ and $d^{+}=-4(a+d+l)+(b+m)^{2}$ and consider the points

$$
\left(x_{0}^{ \pm}, y_{0}\right)=\left(-1,\left(-b+m \pm \sqrt{d^{-}}\right) / 2\right),
$$

and

$$
\left(x_{1}^{ \pm}, y_{1}\right)=\left(1,\left(-b+m \pm \sqrt{d^{+}}\right) / 2\right) .
$$

If $d^{-}>0$ and $d^{+}>0$, then there are four finite hyperbolic singular points: two saddles and two nodes, one stable and other unstable. If $d^{-} d^{+}=0$ and $d^{-}+d^{+}>0$, then there are three finite singular points: a hyperbolic saddle point, a hyperbolic node and a semi-hyperbolic saddle-node point (see Theorem 2.19 of [5]). If $d^{-} d^{+}<0$, then there are two finite hyperbolic singular points, a saddle and a node. If $d^{-}=d^{+}=0$, then there are two semi-hyperbolic singular points, two saddle-node points. If $d^{-} d^{+}=0$ and $d^{-}+d^{+}<0$, then there is one semi-hyperbolic singular point (a saddle-node point). If $d^{-}<0$ and $d^{+}<0$ there are no finite singular points.

Now we consider the study of the invariant straigh lines $x=1$ and $x=-1$ passing through the singular points. Since systems of type $(R P L)$ have the Darboux invariant $I_{1}(x, y, t)=\sqrt{x+1} e^{t} / \sqrt{x-1}$ all orbits of these systems have $\omega$ - and $\alpha$-limit sets contained in the boundary of the Poincaré disc or in the straight lines $x=-1$ and $x=1$.

From the above study we conclude that the local behavior of the finite and infinite singular points of systems of type $(R P L)$ depend on the choice of the parameters $l, m, b$ and $d$ and on the signs of $\delta, d^{+}$and $d^{-}$. Since all possible combinations of the signs of $\delta, d^{+}$and $d^{-}$are realizable, i.e. they occur in systems of the type $(R P L)$ with $n \neq 0$, we obtain the first 18 phase portraits of Figure 1.

Case 2: $n=0$. Under this hypothesis in the chart $U_{1}$ the compactified system of type $(R P L)$ writes

$$
\begin{aligned}
& \dot{z_{1}}=l-z_{1}+m z_{1}+a z_{2}+b z_{1} z_{2}+d z_{2}^{2}+z_{1} z_{2}^{2}, \\
& \dot{z_{2}}=\left(-1+z_{2}\right) z_{2}\left(1+z_{2}\right) .
\end{aligned}
$$

The point $p_{0}=(-l /(m-1), 0)$ is a hyperbolic infinite singular point if $m \neq 1$, it is a saddle if $m>1$, and a node if $m<1$. Moreover, if $m=1$ and $l=0, p_{0}$ does not exist. If $m=1$ and $l \neq 0$ then the two components of the system $(R P L)$ are not coprime, and consequently we do not consider this case.

The system of type $(R P L)$ in the chart $U_{2}$ is

$$
\begin{aligned}
& \dot{z}_{1}=z_{1}^{2}-m z_{1}^{2}-l z_{1}^{3}-b z_{1} z_{2}-a z_{1}^{2} z_{2}-z_{2}^{2}-d z_{1} z_{2}^{2}, \\
& \dot{z_{2}}=-z_{2}\left(m z_{1}+L z_{1}^{2}+b z_{2}+a z_{1} z_{2}+d z_{2}^{2}\right),
\end{aligned}
$$

having the origin as a linearly zero singular point. The local behavior at this point can be studied using the invariant straight lines $x=1$ and $x=-1$ and the separatrices of this system. 
For studying the finite singular point we can proceed as in Case 1 . When $b \neq m$ and $b \neq-m$, the systems of type $(R P L)$ with $n=0$ has two finite hyperbolic singular points $\left(x_{0}, y_{0}\right)=(-1,(a-d-l) /(b-m))$ and $\left(x_{1}, y_{1}\right)=(1,(-a-d-l) /(b+m))$.

The local phase portrait and number of the finite singular points depends on the combinatory of the signs of $b-m$ and $b+m$. If $(b+m)(b-m)>0$, then there are two finite hyperbolic singular points: a saddle and a node. If $(b+m)(b-m)<0$ then there are two hyperbolic finite singular points: two saddles or two nodes (one stable and the other unstable ). If $(b+m)(b-m)=$ 0 and $m \neq 0$ there is only one hyperbolic finite singular point. If $m>0$ then there is a node, if $m<0$ there is a saddle. If $m=b=0$, there is no finite singular point because $d \neq \pm a-l$ (otherwise the two components of the system are not coprime).

Moreover, fixing $(b+m)(b-m)>0$ then $1-m$ could be positive, negative or zero, so all three possible combinatory of the finite and infinite configuration of the singular points could be achievable. The same happens if $b-m<0$ and $b+m>0$, or $b=m>0$. But if $b-m>0$ and $b+m<0$ or $b=m<0$, or $b=m=0$, then $1-m>0$, so there is just one possible configuration for each one. From these arguments we conclude that there are 12 non equivalent phase portraits for systems of type $(R P L)$ when $n=0$. See the phase portraits from 19 to 30 in Figure 1.

Proof of statement $(C P L)$ of Theorem 3. Systems of type $(C P L)$ corresponds to systems (VI) of the article [6]. In that article the study of those systems are divided in six cases. The case (VI.1) provides the phase portraits 1 and 2 of Figure 1 of [6], i.e. the phase portraits 1 and 2 of Figure 2; the case (VI.2) gives the phase portraits 5 and 6 of Figure 1 of [6], i.e. the phase portraits 3 and 4 of Figure 2; the case (VI.3) provides the phase portrait 8 of Figure 1 of [6], i.e. the phase portrait 5 of Figure 2; the case (VI.4) generates the phase portraits 15, 16 and 17 of Figure 1 of [6], i.e. the phase portraits 6,7 and 8 of Figure 2; the case (VI.5) gives the phase portrait 20 of Figure 1 of [6], i.e. the phase portrait 9 of Figure 2. Finally the case (VI.6) provides the phase portraits 21 of Figure 1 of [6], i.e. the phase portrait 10 of Figure 2.

Proof of statement (DL) of Theorem 3. We divide the study of systems of type $(D L)$ in two cases.

Case 1: $n \neq 0$. The study of the infinite singular point of systems of type $(D L)$ coincides with the study of systems of type $(R P L)$. Applying an affine change of variables we can fix $n=1$. In the charts $U_{1}$ and $U_{2}$ the compactified systems of type $(D L)$ with $n=1$ writes exactly as systems of type $(R P L)$, so these systems have at most two hyperbolic infinite singular points

$$
p^{ \pm}=\left(z_{1}, z_{2}\right)=\left(\frac{1}{2}\left(1-m \pm \sqrt{1-4 l-2 m+m^{2}}\right), 0\right) .
$$


Moreover, if we denote $\delta=1-4 l-2 m+m^{2}$ then $\delta>0$ implies that $p^{+}$ is a saddle and $p^{-}$is a node. If $\delta=0$ then $p^{+}=p^{-}$and the unique semihyperbolic infinite singular point at the chart $U_{1}$ is a saddle-node. If $\delta<0$ then there are no infinite singular points in the chart $U_{1}$. The origin of the $U_{2}$ chart is a hyperbolic node.

There are two semi-hyperbolic finite singular points, namely

$$
\left(x_{0}, y_{0}^{ \pm}\right)=\left(0, \frac{1}{2}\left(-b \pm \sqrt{b^{2}-4 d}\right)\right)
$$

if $b^{2}-4 d>0$. It follows from Theorem 2.19 of [5] that both are saddlenodes. If $b^{2}-4 d=0$ then there is one linearly zero singular point. To study this point we need to apply the theory of blow-ups. If $b^{2}-4 d<0$ then there is no finite singular points.

Systems of type $(D L)$ under the hypothesis $b^{2}-4 d=0$ and after translate the singular point to the origin it is writing as

$$
\dot{x}=x^{2}, \quad \dot{y}=a x-\frac{b m}{2} x+l x^{2}+m x y+y^{2} .
$$

After applying the blow-up $x=u, y=u v$ we obtained the system

$$
\dot{u}=u^{2}, \quad \dot{v}=a-\frac{b m}{2}+u\left(l+(-1+m) v+v^{2}\right) .
$$

If $2 a-b m \neq 0$ there is no singular point under the line $u=0$. Otherwise, we need to divide the system by $u$ and the points

$$
\left(u_{0}, v_{0}^{ \pm}\right)=\left(0,\left(1-m \pm \sqrt{1-4 l-2 m+m^{2}}\right) / 2\right)
$$

are singular points. It is easy to conclude that there are two hyperbolic points on $u=0$ if $1-4 l-2 m+m^{2}>0$, or a semi-hyperbolic point if $1-4 l-2 m+m^{2}=0$, which is a saddle-node. Going back through the changes of variables given by the blow-ups we get the local phase portrait of the degenerate singular point $\left(x_{0}, y_{0}\right)$, when $b^{2}-4 d=0$ which is given by two hyperbolic and two parabolic sectors. The localization of these two hyperbolic and parabolic sectors changes when the infinity singular points change their local phase portraits, providing the phase portraits from 4 to 9 of Figure 3.

In short, studying all the possible global connections of the separatrices defined by the local phase portraits of the finite and infinite singular points we get the phase portraits from 1 to 12 of Figure 3.

Case 2: $n=0$. The unique finite singular point is $p_{1}=(0,-d / b)$, if $b \neq 0$. In fact $p_{1}$ is a semi-hyperbolic saddle-node. If $b=0$ there is no finite singular points.

In the chart $U_{1}$ the compactified system of type $(D L)$ writes

$$
\begin{aligned}
& \dot{z_{1}}=l-z_{1}+m z_{1}+a z_{2}+b z_{1} z_{2}+d z_{2}^{2}+z_{1} z_{2}^{2}, \\
& \dot{x_{2}}=\left(-1+z_{2}\right) z_{2}\left(1+z_{2}\right) .
\end{aligned}
$$


So the point $p_{0}=(-l /(m-1), 0)$ is the unique infinite singular point in $U_{1}$ if $m \neq 1$. The point $p_{0}$ is a hyperbolic saddle if $m>1$, and a hyperbolic node if $m<1$. If $m=1$ there is no infinite singular point in the local chart $U_{1}$.

System of type $(D L)$ in the chart $U_{2}$ becomes

$$
\begin{aligned}
& \dot{z}_{1}=z_{1}^{2}-m z_{1}^{2}-l z_{1}^{3}-b z_{1} z_{2}-a z_{1}^{2} z_{2}-d z_{2}^{3}-d z_{1} z_{2}^{2}, \\
& \dot{z}_{2}=-z_{2}\left(m z_{1}+l z_{1}^{2}+b z_{2}+a z_{1} z_{2}+d z_{2}^{2}\right),
\end{aligned}
$$

having the origin as a degenerate singular point. In order to study this point we blow-up it. Doing $z_{1}=u$ and $z_{2}=u v$ we get the system

$$
\begin{aligned}
& \dot{u}=-u\left(-1+m+l u+b v+a u v+d u v^{2}\right), \\
& \dot{v}=-v,
\end{aligned}
$$

which has the origin as the unique singular point when $l^{2}+(m-1)^{2} \neq 0$. If $m<1$ the origin is a hyperbolic saddle, if $m>1$ the origin is a hyperbolic node. In the case where $m=1$ we consider two cases. If $l \neq 0$, then the origin is a semi-hyperbolic saddle-node, and if $l=0$ the line $v=0$ is filled of singular points.

After blow-down we get the local phase portraits of the origin of $U_{2}$ which depends on $m, b$ and $l$. When $m>1$ the origin has two elliptics sectors and parabolic sectors, the distribution of the two elliptic sectors depends on $b$, see phase portrais 13 and 14 of Figure 3. If $m<1$ then there is two hyperbolic sectors and parabolic ones, the distribution of the two hyperbolic sectors depends on $b$, see phase portrais 15 and 16 of Figure 3. If $m=1$ then the origin has an elliptic, a hyperbolic and parabolic sectors when $l b \neq 0$, and it is a stable node (only parabolic sectors) when $l \neq 0$ and $b=0$, see phase portrais 17 and 18 of Figure 3. If $m=1$ and $l=0$ then the infinity is filled of singular points and its local phase portrait depends on $b$, see phase portrais 19 and 20 of Figure 3.

Proof of statement $\left(p_{1}\right)$ of Theorem 3. The parameter $c$ cannot be zero otherwise the two components of the system (4) are not coprime. Then it is easy to check that the origin is a focus if $r \neq 0$ and a node if $r=0$. The system at infinity has only a pair of singular points, such that using the Theorem 2.19 of [5], they are saddle-nodes having the two hyperbolic sectors at the same side of the straight line of the infinity. Since the existence of the Darboux invariant prevents the existence of limit cycles (see Proposition 5) we get that the phase portrait of system (4) is the one of Figure 4.

Proof of statement $\left(p_{2}\right)$ of Theorem 3. In order to study system (5) we divide the study in three cases.

Case 1: $r a b(1-v) \neq 0$. Then system (5) admites two finite singular points. They are $\left(x_{0}, y_{0}\right)=(0,0)$ and $\left(x_{1}, y_{1}\right)=((2 r+c v) /(a(1-v)),(c+2 r) /(b(v-$ $1))$. The origin is a focus. Indeed the eigenvalues of this singular point are 
$c \pm i r$, then the origin is a focus when $c \neq 0$, and a weak focus or a center when $c=0$. In this last case as system (5) admits Darboux invariant given by (6) if follows from Proposition 5 that the origin is a focus when $c=0$. The point $\left(x_{1}, y_{1}\right)$ is a saddle point if $a b(v-1)<0$, and a node if $a b(v-1)>0$.

At infinity there is a unique singular point in the local chart $U_{1}$ given by $\left(z_{1}, 0\right)=(-a / b v, 0)$ when $v \neq 0$ and this point is a when $a b(v-1)>0$ and a node if $a b(v-1)<0$. When $v=0$ there is no singular point in $U_{1}$. At the local chart $U_{2}$, there is no singular point under the condition $v \neq 0$, and the origin is a singular point if $v=0$. The origin of $U_{2}$ is a saddle point if $a b>0$ and a node if $a b<0$. From this study we obtain the phase portraits 1 and 2 of Figure 5.

Case 2: $r \neq 0$ and $a b(v-1)=0$. Then the origin is the unique finite singular point of system (5) and from the reasons given in Case 1 it is a focus except when $r=0$. Now we study the singular points at infinity. We distinguish three subcases.

Subcase 2.1: $a=0$. Since $a^{2}+b^{2} \neq 0$ this implies that $b \neq 0$. Then there is a unique singular point at infinity which is the $(0,0)$ of the local chart $U_{1}$. This singular point is semi-hyperbolic and its study shows that it is a saddle-node having the two hyperbolic sectors at the same side of the infinity.

Subcase 2.2: $b=0$. Consequently $a \neq 0$. The study is similar to the Subcase 2.1 and we get the same kind of saddle-node but this time at the origin of the local chart $U_{2}$.

Subcase 2.3: $v=1$. Again there is a unique semi-hyperbolic saddle-node with two hyperbolic sectors at the same side of the infinity.

Finally taking in account these local behaviors at the finite and infinite singular points we get the phase portrait 3 of Figure 5 .

Case 3: $r=0$. We distinguish two subcases.

Subcase 3.1: $c \neq 0$. Then the study of this subcase is the complete similar to the study of the Case 1 but now the origin is a node instead of a focus. But topologically the phase portraits in this case are also given by the phase portraits 1 and 2 in Figure 5.

Subcase 3.2: $c=0$. Then the system (5) is a homogeneous system of degree 2 and it follows from [4] that the phase portrait is given by the phase portrait 4 of Figure 5.

\section{ACKNowledGements}

The first author is partially supported by the grants MICINN/FEDER MTM 2008-03437, AGAUR 2009SGR 410, ICREA Academia and by FP7PEOPLE-2012-IRSES numbers 318999. Both authors are also supported by the joint project CAPES-MECD grant PHB-2009-0025-PC and by FP7PEOPLE-2012-IRSES numbers 316338. 


\section{REFERENCES}

[1] Y. Bolaños, J. Llibre AND C.VAlls, Phase portraits of quadratic Lotka-Volterra Systems with a Darboux invariant in the Poincare disc, to appear in Communications in Contemporary Mathematics.

[2] L. CAIRó AND J. Llibre, Darbouxian first integrals and invariants for real quadratic systems having an invariant conic, J. Phys. A: Math. Gen. 35 (2002), 589 - 608.

[3] C. J. Christopher And J. Llibre, Integrability via invariant algebraic curves for planar polynomial differential systems, Ann. Differ. Equ. 16 (2000), 5-19.

[4] T. DATE, Classification and analysis of two-dimensional real homogeneous quadratic differential equation systems, J. Differential Equations 32 (1979) 311-334.

[5] F. Dumortier, J. Llibre And J. C. Artés, Qualitative theory of planar differential systems, Universitext, Springer, New York, 2006.

[6] A. Gasull, S. Li-Ren And J. Llibre, Chordal quadratic system, Rocky Mountain J. Math.16 (1986), 751-781.

[7] J. W. REYN, A bibliography of the qualitative theory of quadratic systems of differential equations in the plane, Delf University of Technology, http://ta.twi.tudelf.nl/DV/Staff/ J.W.Reyn.html, 1997.

Departament de Matemàtiques, Universitat Autònoma de Barcelona, 08193 Bellaterra, Barcelona, Catalonia, Spain

E-mail address: jllibre@mat.uab.cat

Departamento de Matemática, Instituto de Ciências Matemáticas e ComputaÇão, Universidade de SÃo Paulo, C.P 668, 13.560-970, SÃo CArlos, SP, Brazil.

E-mail address: regilene@icmc.usp.br 